\title{
Measures to Reduce X-ray Noise in a CCD High Quality Image Camera for HVEM
}

\author{
Kiyokazu Yoshida*, Tadao Furutsu**, Shinji Shimojo*** and Hirotaro Mori* \\ *Research Center for Ultra-high Voltage Electron Microscopy, Osaka University, 2-1, Yamada-oka, \\ Suita, Osaka 565-0871 Japan, \\ **BTC, Naka Division, Hitachi High-Technologies Co., Hitachinaka, Ibaragi 312-0033 Japan \\ ***Cybermedia Center, Osaka University, 5-1, Mihogaoka, Ibaraki, Osaka 567-0047 Japan
}

In order to construct a system with which high quality still images of the $3 \mathrm{MV}$ high voltage electron microscope (HVEM) can be recorded and, upon request, be sent to a remote laboratory, a $4096 \times$ 4096 (i.e. 16M) pixel high resolution CCD camera was attached to the 3MV HVEM,. With this $16 \mathrm{M}$ CCD camera, it is possible to record a large amount of picture information comparable to that in negative films. A characteristic subject to be settled with the CCD camera for HVEM is the way to reduce the noise by X-rays. When a high energy X-ray quatum penetrates an element of the detector, a pixel corresponding to the element appears as a white spot. As a result of accumulation of such events, there appear at random a high density of white spots in an electron microscope image. An example of random noise is depicted in an enlarged portion (inset) of an electron micrograph shown in Fig.1, which is a cross-sectional view of a modern LSI device. Five measures against Xray noise have been taken in the present work.

(1)Lead blocks: A general measure might be to enclose the camera with lead blocks to shield it from X-rays. However, this way is not realistic for HVEM since lead blocks thicker than at least $100 \mathrm{~mm}$ (being very heavy) are necessary to shield $\mathrm{MeV}$ X-ray quanta and, on top of that, it is impossible to close the optical incidence port in front of the CCD unit during the observation. Based upon this premise, instead of enclosing the whole camera with a huge lead block, use of small, 50mm thick blocks, being compatible with the construction from the weight view point, has been employed as shield blocks. (2)SA aperture: Fig.2(a) to (c) show images of a fixed illuminated area of a standard amorphous carbon film with shadows of SA aperture with different sizes, whereas Fig2(a') to (c') show enlarged images of the framed portion in Fig.2(a) to (c), respectively. It is evident from Fig.2 that the areal density of the white spots can be greatly reduced with decreasing size of SA aperture. This fact suggests that with decreasing size of SA aperture the number of electrons colliding with the inner wall of microscope column decreases rapidly, resulting in the effective suppression of X-ray emission inside the column. Thus it is confirmed that the control of SA aperture size is an effective way to reduced the X-ray noise. So, in the present work, a special aperture equivalent to the SA aperture has been set at a position just below the projection lens.

(3)Mirror: Fig.3 is a schematic illustration of the present camera module. Optical images formed on the fluorescent screen are transferred via a mirror and a lens to the detector of the CCD unit. A mirror to bend the optical axis by 90 degrees was employed to avoid any direct incidence of X-ray quanta into the CCD unit. Conventional mirror is made of a $1 \mathrm{~mm}$-thick quartz plate (substrate) vapor-deposited with aluminum. However, it is known that the quartz plate acts as a strong X-ray emitter. Therefore, in the present work, a Kapton film of $25 \mu \mathrm{m}$ in thickness has been employed as a substrate material for the mirror. (4)Bench for mirror: In general, bench for mirror also acts as an emitter of X-rays. Therefore, use of a slim bench is preferred in view of reducing X-ray noise. Conventional bench for mirror is fat and voluminous (Fig.4(a)). This is because it should support a rather heavy mirror made of quartz substrate. In the present design, however, the bench can be made much slim since the mirror becomes much light due to the employment of the Kapton substrate. Based upon this premise, a slim bench such as depicted in Fig. 4(b) has been constructed and used in the present camera module. (5)Lead glass: One or two pieces of $19 \mathrm{~mm}$ thick lead glass $(50 \%$ 
equivalent) has been placed in front of the optical lens (Fig.3) of the camera module to reduce the entrance of X-ray quanta. The lead glass stops also optical photons, so an optimum thickness of glass has been searched out by monitoring the image quality.

Effects of measures (1),(3), and (5) mentioned above, have been examined in an accumulative manner by an experiment shown in Fig.5. Fig.5(a) shows an image of an area without any measures, Fig.5(b) an image of the same area with measure (1), Fig.5(c) with measures (1) and (3), Fig.5(d) with measures (1), (3) and (5) [one piece of glass] and Fig.5(e) with (1), (3) and (5) [two pieces of glass], respectively. (Each micrograph in Fig.5 is of $500 \times 1000$ pixel, which is cut out from original $4096 \times 4096$ pixel image.) It is evident from Fig.5 that with accumulation of these measures, the areal density of white spots is remarkably decreased. The noise level in Fig.5(e) corresponds to a level of white noise from the fluorescent material itself. At such a reduced level as shown in

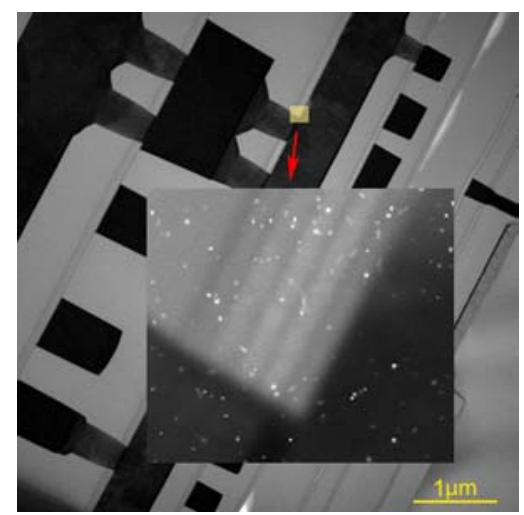

Fig 1. An example of random noise by X-rays in an electronmicrograph taken at $2 \mathrm{MV}$.

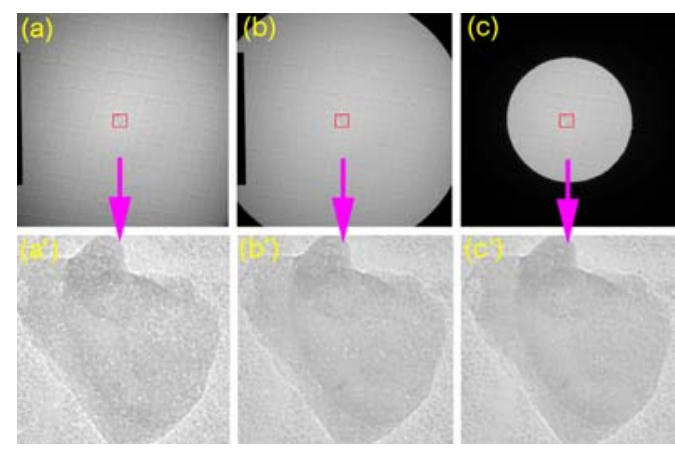

Fig,2 Effect of SA aperture size on X-ray noise. software. Fig.5(e), it seems that further elimination of the noise may be effectively and therefore preferably done by using an appropriate

\section{Acknowledgements}

This work was in part supported by the Ministry of Education, Science, and Culture under a Grant-in-Aid for Scientific Research (Grant No.13224059), and in part by

"Nanotechnology Support Project" of the Ministry of Education, Culture, Sports, Science and Technology (MEXT), Japan.

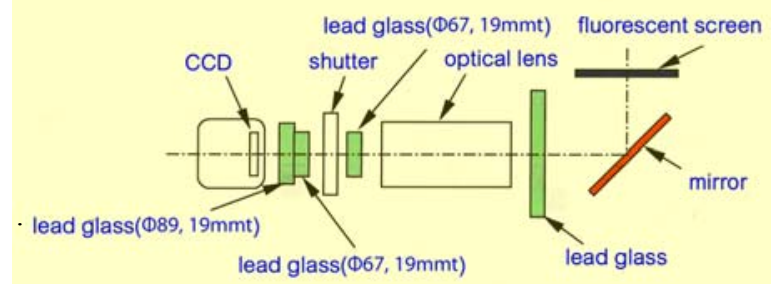

Fig.3 Schematic illustration of the present camera module.
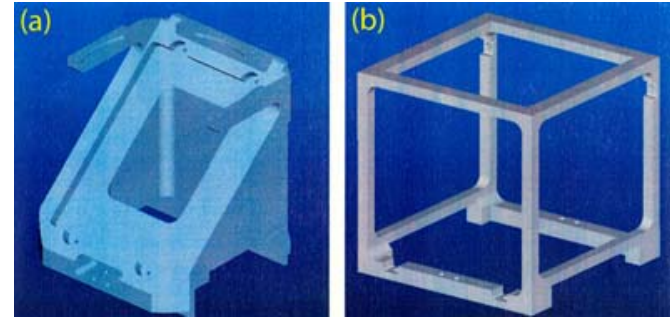

Fig.4 Benches for mirror. (a)conventional, (b)present design.
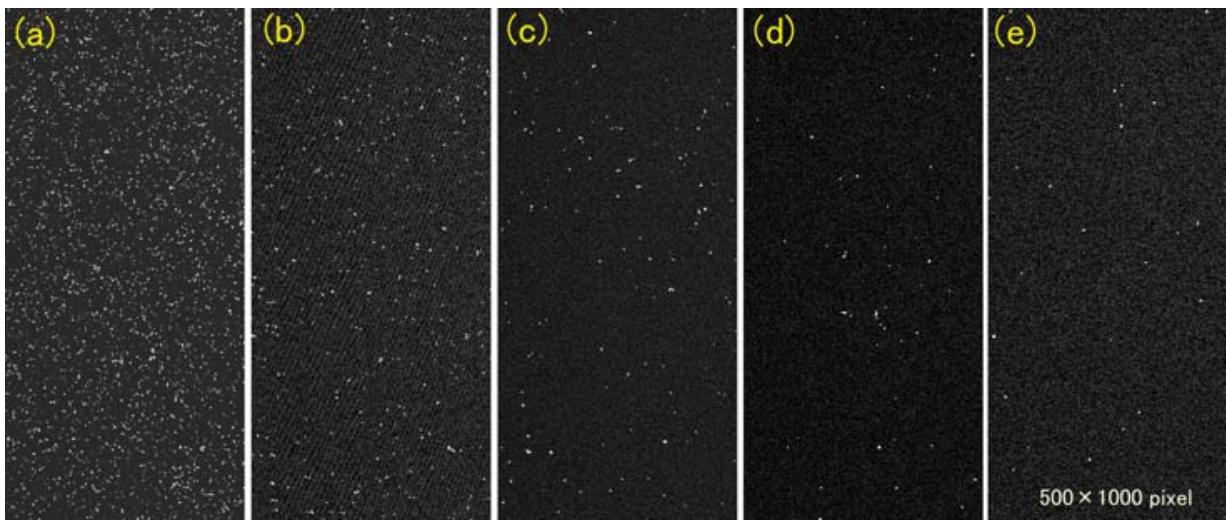

Fig. 5 Effect of measures employed in the present design. 